\title{
SHAPES OF STATIONARY AUTOCOVARIANCES
}

\author{
ROBERT LUND, ${ }^{* * *}$ Clemson University \\ YING ZHAO, ${ }^{* * *}$ University of Georgia \\ PETER C. KIESSLER, ${ }^{*}$ Clemson University
}

\begin{abstract}
This note introduces shape orderings for stationary time series autocorrelation and partial autocorrelation functions and explores some of their convergence rate ramifications. The shapes explored include decreasing hazard rate and new better than used, orderings that are familiar from stochastic processes settings. Time series models where these shapes arise are presented. The shapes are used to obtain explicit geometric convergence rates for mean squared errors of one-step-ahead forecasts.
\end{abstract}

Keywords: ARMA process; autocorrelation; convergence rate; log-convexity; mean squared error; NWU; partial autocorrelation

2000 Mathematics Subject Classification: Primary 60G10

Secondary 60G20

\section{Introduction}

Stochastic shape orderings for the autocorrelation (ACF) and partial autocorrelation (PACF) functions of stationary time series are introduced and explored here. The orderings examined include new worse than used, new better than used, decreasing hazard rate, and increasing hazard rate. The utility of such orderings and their variants is well known in stochastic processes (see Brown (1980), Shaked and Shanthikumar (1994), Liggett (1989), Hansen and Frenk (1991), Kijima (1997), Berenhaut and Lund (2001), (2002), Müller and Stoyan (2002), and Lund et al. (2006), amongst others) and appears to be a promising addition to the time series analyst's toolbox. We are unaware of any previous literature on shapes of time series.

\section{Definition of orderings}

Let $\left\{X_{t}\right\}$ be a zero-mean stationary series with autocovariance function (ACVF) $\gamma(h)=$ $\operatorname{cov}\left(X_{t+h}, X_{t}\right)$ at lag $h$. We denote the lag- $h$ autocorrelations and partial autocorrelations by $\rho(h)=\operatorname{corr}\left(X_{t+h}, X_{t}\right)$ and $\alpha(h)=\operatorname{corr}\left(X_{h+1}, X_{1} \mid X_{2}, X_{3}, \ldots, X_{h}\right)$, respectively. To clarify, a conditional correlation at lag $h \geq 2$ refers to correlation or residuals after best linear prediction from $X_{2}, \ldots, X_{h}$, i.e.

$$
\alpha(h)=\operatorname{corr}\left(X_{h+1}-\mathrm{P}\left(X_{h+1} \mid X_{2}, \ldots, X_{h}\right), X_{1}-\mathrm{P}\left(X_{1} \mid X_{2}, \ldots, X_{h}\right)\right),
$$

where $\mathrm{P}\left(Y \mid Z_{1}, \ldots, Z_{k}\right)$ denotes the best prediction of $Y$ from linear combinations of $Z_{1}, \ldots, Z_{k}$.

Received 6 October 2004; revision received 13 September 2006.

* Postal address: Department of Mathematical Sciences, Clemson University, Clemson, SC 29634-0975, USA.

** Email address: lund@ces.clemson.edu

*** Current address: 2-2-502 Qingfeng Huajing Yuan, Haidian Qu, Beijing, 100085, P. R. China. 
The $\operatorname{ACF} \rho(\cdot)$ is said to be new better than used if

$$
\rho(i+j) \leq \rho(i) \rho(j), \quad i, j \geq 0,
$$

and is said to be new worse than used if

$$
\rho(i+j) \geq \rho(i) \rho(j), \quad i, j \geq 0 .
$$

The ACF $\rho(\cdot)$ is said to be log-convex if

$$
\rho(h+1)^{2} \leq \rho(h) \rho(h+2), \quad h \geq 0,
$$

and is said to be log-concave if

$$
\rho(h+1)^{2} \geq \rho(h) \rho(h+2), \quad h \geq 0 .
$$

Log-convexity is also called decreasing hazard rate and log-concavity is also called increasing hazard rate, though the term increasing hazard rate is preferably used when $\rho(\cdot)$ can be negative (hence, not permitting a logarithm), which is allowed here. The acronyms NWU, NBU, DHR, and IHR are used for new worse than used, new better than used, decreasing hazard rate, and increasing hazard rate, respectively.

We say that $\left\{X_{t}\right\}$ has a monotone autocorrelation function if $\rho(h)$ is nonincreasing with increasing $h$. A stationary autocorrelation $\rho(\cdot)$ cannot be monotone increasing since $|\rho(h)| \leq 1$ for all $h \geq 1$ and $\rho(0)=1$. Finally, $\left\{X_{t}\right\}$ is said to have a convex ACF if $\rho(h)$ is convex in $h$, i.e.

$$
\rho(h)-2 \rho(h-1)+\rho(h-2) \geq 0, \quad h \geq 2 .
$$

As many convex sequences with $\rho(0)>0$ are nonnegative definite (this is Polya's criterion), such sequences are legitimate stationary ACFs (Billingsley (1995, Problem 26.3) provided sufficient conditions). Not every stationary ACF satisfies (2.5):

$$
\rho(h)= \begin{cases}\left(\frac{1}{2}\right)^{h / 2} & \text { for } h \geq 0 \text { even } \\ 0 & \text { otherwise }\end{cases}
$$

is one such example.

The above shapes apply equally well to the ACVF and PACF; we just replace $\rho(h)$ with $\gamma(h)$ and $\alpha(h)$, respectively. We will not, however, attempt to attach meaning to NBU and NWU ACVFs as this would lead to inconsistent units on the two sides of (2.1) and (2.2) (correlations are unitless but autocovariances are not).

\section{Some general results}

Consider a causal linear process

$$
X_{t}=\sum_{k=0}^{\infty} \psi_{k} Z_{t-k},
$$

where $\left\{Z_{t}\right\}_{t=-\infty}^{\infty}$ is zero-mean white noise with variance $\sigma^{2}$ and $\sum_{k=0}^{\infty}\left|\psi_{k}\right|<\infty$. Causal linear processes comprise a large class of stationary processes. Any ARMA process whose autoregressive polynomial is root-free on and inside the unit circle, for example, can be expressed as in (3.1); see Brockwell and Davis (1991, Chapter 3). 
Theorem 3.1. Consider the causal linear process in (3.1).

(a) If $\psi_{k}$ is nonnegative and nonincreasing in $k$, then $\gamma(h)$ is nonnegative and nonincreasing in $h$.

(b) If $\psi_{k}$ is nonnegative and convex in $k$, then $\gamma(h)$ is nonnegative and convex in $h$.

(c) If $\psi_{k}$ is nonnegative and DHR in $k$, then $\gamma(h)$ is nonnegative and DHR in $h$.

(d) If $\left\{X_{t}\right\}$ is the unique (in mean square) solution to the causal ARMA equation

$$
X_{t}-\sum_{k=1}^{p} \phi_{k} X_{t-k}=Z_{t}+\sum_{k=1}^{q} \theta_{k} Z_{t-k},
$$

and the coefficients $\phi_{1}, \ldots, \phi_{p}$ and $\theta_{1}, \ldots, \theta_{q}$ are nonnegative, then $\gamma(h) \geq 0$ for all $h \geq 1$.

Proof. The autocovariance $\gamma(h)$ is obtained from (3.1) as follows:

$$
\gamma(h)=\sigma^{2} \sum_{k=0}^{\infty} \psi_{k} \psi_{k+h} .
$$

Hence, the nonnegativity assertions in parts (a)-(c) follow. The monotonicity in part (a) is established by using (3.3) and $\psi_{k+h+1} \leq \psi_{k+h}$. Part (b) follows from (3.3) and the convex structure assumed on $\left\{\psi_{k}\right\}$. Part (c) is proven using (2.3) and the Cauchy-Schwarz inequality, i.e. for each $h \geq 0$, we obtain

$$
\begin{aligned}
\gamma(h+1) & =\sigma^{2} \sum_{k=0}^{\infty} \psi_{k} \psi_{k+h+1} \\
& \leq \sigma^{2} \sum_{k=0}^{\infty} \sqrt{\psi_{k} \psi_{k+h}} \sqrt{\psi_{k} \psi_{k+h+2}} \\
& \leq \sqrt{\sigma^{2} \sum_{k=0}^{\infty} \psi_{k} \psi_{k+h}} \sqrt{\sigma^{2} \sum_{k=0}^{\infty} \psi_{k} \psi_{k+h+2}} \\
& =\sqrt{\gamma(h) \gamma(h+2)} .
\end{aligned}
$$

Part (d) is established by showing that $\psi_{k} \geq 0$ for each $k \geq 0$. This can be done inductively with the ARMA recursion for $\psi_{j}$, with $\psi_{0}=1$ and

$$
\psi_{j}=\sum_{k=1}^{\min (p, j)} \phi_{k} \psi_{j-k}+\mathbf{1}_{\{j \leq q\}} \theta_{j}, \quad j \geq 1,
$$

where $\mathbf{1}_{\{\cdot\}}$ is the indicator function. (Equation (3.4) is Equations (3.3.3) and (3.3.4) from Brockwell and Davis (1991).)

Turning to shapes of common time series models, we offer the following structure on secondorder autoregressions $(\operatorname{AR}(2))$. Here, the series $\left\{X_{t}\right\}$ is the unique (in mean square) solution to the second-order difference equation $X_{t}-\phi_{1} X_{t-1}-\phi_{2} X_{t-2}=Z_{t}$, where $\left\{Z_{t}\right\}$ is zero-mean white noise with variance $\sigma^{2}$. 
Theorem 3.2. Suppose that the roots of the autoregressive polynomial $1-\phi_{1} z-\phi_{2} z^{2}$ lie outside the unit circle (the model is causal) and are complex conjugates or repeated real roots. Then the ACF of this series is log-concave (IHR).

Proof. Expressing the AR(2) polynomial in terms of its roots $\xi_{1}$ and $\xi_{2}$ gives

$$
\left(1-\xi_{1}^{-1} B\right)\left(1-\xi_{2}^{-1} B\right) X_{t}=Z_{t},
$$

where $B$ is the usual backshift operator and causality implies that $\left|\xi_{1}\right|>1$ and $\left|\xi_{2}\right|>1$. The roots and $\operatorname{AR}(2)$ parameters are related by $\phi_{1}=\xi_{1}^{-1}+\xi_{2}^{-1}$ and $\phi_{2}=-\xi_{1}^{-1} \xi_{2}^{-1}$. Equation (3.3.14) in Brockwell and Davis (1991) identifies the following form for the ACVF:

$$
\gamma(h)=\frac{\sigma^{2} \xi_{1}^{2} \xi_{2}^{2}}{\left(\xi_{1} \xi_{2}-1\right)\left(\xi_{2}-\xi_{1}\right)}\left[\left(\xi_{1}^{2}-1\right)^{-1} \xi_{1}^{1-h}-\left(\xi_{2}^{2}-1\right)^{-1} \xi_{2}^{1-h}\right] .
$$

For the case of complex conjugate roots, we write $\xi_{1}=r \mathrm{e}^{\mathrm{i} \theta}$ and $\xi_{2}=r \mathrm{e}^{-\mathrm{i} \theta}$ for some $\theta \in(0, \pi]$ and $r>1$ in (3.5), to get

$$
\gamma(h)=\frac{\sigma^{2} r^{4} r^{-h} \sin (h \theta+\psi)}{\left(r^{2}-1\right)\left(r^{4}-2 r^{2} \cos (2 \theta)+1\right)^{1 / 2} \sin \theta},
$$

where $\tan \psi=\left(r^{2}+1\right)\left(r^{2}-1\right)^{-1} \tan \theta$ and $\cos \psi$ has the same sign as $\cos \theta$. Hence,

$$
\rho(h)=\frac{r^{-h} \sin (h \theta+\psi)}{\sin \psi}=\left(\frac{\sin (h \theta)}{\tan \psi}+\cos (h \theta)\right) r^{-h} .
$$

Some tedious but straightforward manipulations of (3.7) now give

$$
\rho^{2}(h+1)-\rho(h) \rho(h+2)=\left(\frac{1}{2}+\frac{1}{2 \tan ^{2} \psi}\right)(1-\cos (2 \theta)) r^{-2(h+1)} .
$$

As $1-\cos (2 \theta) \geq 0, r>1$, and $\tan ^{2} \psi \geq 0$, the result now follows for the case of complex conjugate roots. With repeated real roots (i.e. $\xi_{1}=\xi_{2}$ ), the form of (3.6) is $\rho(h)=A_{0} \xi_{1}^{-h}+$ $A_{1} h \xi_{1}^{-h}$, for some constants $A_{0}$ and $A_{1}$, which satisfies

$$
\rho^{2}(h+1)-\rho(h) \rho(h+2)=A_{1}^{2}\left(\xi_{1}^{2}\right)^{-(h+1)}
$$

and is, hence, IHR.

When the AR(2) polynomial is causal and has two distinct real roots, the ACVF will be IHR whenever the product of the two roots, $\xi_{1} \xi_{2}$, is positive (or, equivalently, when $\phi_{2}<0$ ). In the event that $\xi_{1} \xi_{2}$ is negative, the ACVF will be neither IHR or DHR. However, in all cases, the subsequence $\{\gamma(2 h)\}_{h=0}^{\infty}$ can be shown to be IHR.

We close this section with an example of a log-convex (DHR) PACF.

Proposition 3.1. A causal and invertible $\operatorname{ARMA}(1,1)$ series has a PACF whose square is monotonically decreasing and is DHR.

Proof. The $\operatorname{ARMA}(1,1)$ difference equation is

$$
X_{t}-\phi X_{t-1}=Z_{t}+\theta Z_{t-1}
$$


where $\left\{Z_{t}\right\}$ is zero-mean white noise with variance $\sigma^{2}$. Causality and invertibility imply that $|\phi|<1$ and $|\theta|<1$.

The partial autocorrelation function of an $\operatorname{ARMA}(1,1)$ series can be explicitly identified from the result of Problem 5.13 in Brockwell and Davis (1991) as follows:

$$
\alpha^{2}(n)=\frac{\theta^{2 n-2}\left(1-\theta^{2}\right)^{2}(\theta+\phi)^{2}(1+\theta \phi)^{2}}{\left[(\theta+\phi)^{2}\left(1-\theta^{2 n}\right)+\left(1-\phi^{2}\right)\left(1-\theta^{2}\right)\right]^{2}} .
$$

The claimed monotonicity follows from (3.9) since

$$
\begin{aligned}
\frac{\alpha^{2}(n-1)}{\alpha^{2}(n)} & =\frac{1}{\theta^{2}} \frac{\left[(\theta+\phi)^{2}\left(1-\theta^{2 n}\right)+\left(1-\phi^{2}\right)\left(1-\theta^{2}\right)\right]^{2}}{\left[(\theta+\phi)^{2}\left(1-\theta^{2 n-2}\right)+\left(1-\phi^{2}\right)\left(1-\theta^{2}\right)\right]^{2}} \\
& \geq \frac{1}{\theta^{2}} \\
& \geq 1
\end{aligned}
$$

which implies that $\alpha^{2}(n)$ is nonincreasing in $n$. The DHR structure of $\alpha^{2}(\cdot)$ follows from the fact that $\alpha^{2}(n-1) / \alpha^{2}(n)$ is decreasing in $n$, which can be verified from (3.9).

\section{Convergence rates}

As a short application, this section briefly explores convergence rate consequences of the above orderings in one-step-ahead linear prediction settings. This topic was also studied in Pourahmadi (2001, Section 7.6.2). We assume here that $\left\{X_{t}\right\}$ is stationary with zero mean and has $\operatorname{ACVF} \gamma(\cdot), \operatorname{ACF} \rho(\cdot)$, and PACF $\alpha(\cdot)$.

Let $\hat{X}_{t+1}=\mathrm{P}\left(X_{t+1} \mid X_{1}, \ldots, X_{t}\right)$ be the best one-step-ahead prediction of $X_{t+1}$ from linear combinations of $X_{1}, \ldots, X_{t}$ and let $v_{t}=\mathrm{E}\left[\left(X_{t+1}-\hat{X}_{t+1}\right)^{2}\right]$ denote the unconditional mean squared error of this prediction. Let $v_{\infty}=\lim _{t \rightarrow \infty} v_{t}$ denote the limiting mean squared prediction error; this limit exists as $v_{t}$ is nonincreasing in $t$. The mean squared prediction error can be expressed, via the square of the PACF, as

$$
v_{t}=\gamma(0) \prod_{j=1}^{t}\left(1-\alpha^{2}(j)\right)
$$

see Brockwell and Davis (1991, Proposition 5.2.1) and Pourahmadi (2001, Section 7.5). To avoid trivialities, we assume that the covariance matrix of $\left(X_{1}, \ldots, X_{n}\right)^{\prime}$ is invertible for all positive integers $n$ (otherwise, we would have perfect prediction). A sufficient condition for this is merely that $\gamma(h) \rightarrow 0$ as $h \rightarrow \infty$ (see Brockwell and Davis (1991, Proposition 5.1.1)), as is the case for any causal $\operatorname{ARMA}(p, q)$ series.

From (4.1), we deduce that

$$
\begin{aligned}
\left|v_{t}-v_{\infty}\right| & =\gamma(0) \prod_{j=1}^{t}\left(1-\alpha^{2}(j)\right)\left[1-\prod_{j=t+1}^{\infty}\left(1-\alpha^{2}(j)\right)\right] \\
& \leq \gamma(0) \prod_{j=1}^{t}\left(1-\alpha^{2}(j)\right) \sum_{j=t+1}^{\infty} \alpha^{2}(j)
\end{aligned}
$$


where the inequality $1-\prod_{j=t+1}^{\infty}\left(1-\alpha^{2}(j)\right) \leq \sum_{j=t+1}^{\infty} \alpha^{2}(j)$ has been applied. Note that (4.2) is tight for an autoregression of order $p$; specifically, the right-hand side of (4.2) is zero for lags $t>p$, implying that $v_{t} \equiv \sigma^{2}=v_{\infty}$ for $t>p$.

Now consider the case where $\left\{X_{t}\right\}$ has a nonnegative and NBU PACF. Then $\alpha(t+h) \leq$ $\alpha(t) \alpha^{h}(1)$ for all $t, h \geq 0$. Using this in (4.2) produces a very clean and explicit convergence bound,

$$
\left|v_{t}-v_{\infty}\right| \leq \gamma(0) \alpha^{2}(1)\left[\prod_{j=2}^{t}\left(1-\alpha^{2}(j)\right)\right] \alpha^{2}(t) \leq \gamma(0) \alpha^{2}(t)
$$

where $0 \leq \prod_{j=2}^{t}\left(1-\alpha^{2}(j)\right) \leq 1$ and $\alpha^{2}(1) \leq 1$ have been applied.

For bounds with a different shape, suppose that $\alpha^{2}(\cdot)$ is DHR (as in Proposition 3.1). By monotonicity of $\alpha^{2}(n) / \alpha^{2}(n-1)$, we define $M_{\infty}=\lim _{n \rightarrow \infty} \alpha^{2}(n) / \alpha^{2}(n-1)$. Then we obtain

$$
\begin{aligned}
\sum_{j=t+1}^{\infty} \alpha^{2}(j) & =\frac{\alpha^{2}(t+1)}{\alpha^{2}(t)} \alpha^{2}(t)+\frac{\alpha^{2}(t+2)}{\alpha^{2}(t+1)} \frac{\alpha^{2}(t+1)}{\alpha^{2}(t)} \alpha^{2}(t)+\cdots \\
& \leq \alpha^{2}(t)\left[M_{\infty}+M_{\infty}^{2}+\cdots\right] \\
& =\frac{M_{\infty} \alpha^{2}(t)}{1-M_{\infty}}
\end{aligned}
$$

if $M_{\infty}<1$. Substituting (4.3) into (4.2) gives

$$
\left|v_{t}-v_{\infty}\right| \leq \gamma(0) \frac{M_{\infty} \alpha^{2}(t)}{1-M_{\infty}} .
$$

Other shapes could lead to different inequalities; the theme is simply that a shape constraint can provide clean explicit convergence rates of the mean squared prediction errors to their limit.

Explicit convergence bounds for other forecasting quantities can also be obtained via shapes. Elaborating on this, suppose that $\left\{X_{t}\right\}$ is a causal and invertible $\operatorname{ARMA}(p, q)$ series satisfying (3.2). The innovations one-step-ahead recursive prediction equation is

$$
\hat{X}_{n+1}=\sum_{k=1}^{p} \phi_{k} X_{n+1-k}+\sum_{k=1}^{q} \theta_{n, k}\left(\hat{X}_{n+1-k}-X_{n+1-k}\right), \quad n \geq \max (p, q) .
$$

The $\theta_{n, k}$ are defined as

$$
\theta_{n, k}=v_{n-k}^{-1} \mathrm{E}\left[X_{n+1}\left(X_{n+1-k}-\hat{X}_{n+1-k}\right)\right], \quad 1 \leq k \leq q, n \geq \max (p, q) ;
$$

see Brockwell and Davis (1991, Chapter 5). It is also known that $\theta_{n, k} \rightarrow \theta_{k}$ as $n \rightarrow \infty$. In our final result we derive an explicit bound for this convergence in the case of an $\operatorname{ARMA}(1,1)$ model.

Proposition 4.1. The causal and invertible ARMA(1,1) difference equation (3.8) has $\theta_{n, 1} \rightarrow$ $\theta$ with explicit rate

$$
\left|\theta_{n, 1}-\theta\right| \leq \sqrt{2}|\theta|^{n}+|\theta|^{2 n-1} \frac{(1+\phi \theta)^{2}}{\left(1-\phi^{2}\right)\left(1-\theta^{2}\right)} \leq \frac{6|\theta|^{n-1}}{\left(1-\phi^{2}\right)\left(1-\theta^{2}\right)},
$$

for $n \geq 1$. 
Proof. Write the ARMA(1, 1) model as in (3.8). Equation (4.5) is

$$
\hat{X}_{n+1}-\phi X_{n}=\theta_{n, 1}\left(X_{n}-\hat{X}_{n}\right), \quad n \geq 1 \text {. }
$$

Multiplying both sides of (4.7) by $\hat{X}_{n}-X_{n}$ and taking expectations gives

$$
\theta_{n, 1}=\frac{\mathrm{E}\left[\left(\hat{X}_{n+1}-\phi X_{n}\right)\left(X_{n}-\hat{X}_{n}\right)\right]}{v_{n-1}} .
$$

The orthogonality of $X_{n}-\hat{X}_{n}$ and $X_{n+1}-\hat{X}_{n+1}$ and (3.8) provide

$$
\begin{aligned}
v_{n-1} & \theta_{n, 1} \\
& =\mathrm{E}\left[\left(X_{n+1}-\phi X_{n}\right)\left(X_{n}-\hat{X}_{n}\right)\right] \\
& =\mathrm{E}\left[X_{n+1}\left(X_{n}-\hat{X}_{n}-Z_{n}\right)\right]+\mathrm{E}\left[X_{n+1} Z_{n}\right]-\phi \mathrm{E}\left[X_{n}\left(X_{n}-\hat{X}_{n}-Z_{n}\right)\right]-\phi \mathrm{E}\left[X_{n} Z_{n}\right] \\
& =\mathrm{E}\left[\left(X_{n+1}-\phi X_{n}\right)\left(X_{n}-\hat{X}_{n}-Z_{n}\right)\right]+\theta \sigma^{2} .
\end{aligned}
$$

This gives the following equation for $\theta_{n, 1}-\theta$ :

$$
\theta_{n, 1}-\theta=\frac{\mathrm{E}\left[\left(X_{n+1}-\phi X_{n}\right)\left(X_{n}-\hat{X}_{n}-Z_{n}\right)\right]+\theta\left(\sigma^{2}-v_{n-1}\right)}{v_{n-1}} .
$$

To bound quantities in (4.8), the Cauchy-Schwarz inequality provides

$$
\begin{aligned}
\left|\theta_{n, 1}-\theta\right| & \leq \frac{\operatorname{var}\left(X_{n+1}-\phi X_{n}\right)^{1 / 2} \mathrm{E}\left[\left(X_{n}-\hat{X}_{n}-Z_{n}\right)^{2}\right]^{1 / 2}}{v_{n-1}}+\frac{|\theta|\left(v_{n-1}-\sigma^{2}\right)}{v_{n-1}} \\
& =\frac{\sigma\left(1+\theta^{2}\right)^{1 / 2} \mathrm{E}\left[\left(X_{n}-\hat{X}_{n}-Z_{n}\right)^{2}\right]^{1 / 2}}{v_{n-1}}+\frac{|\theta|\left(v_{n-1}-\sigma^{2}\right)}{v_{n-1}} .
\end{aligned}
$$

A bound for $\mathrm{E}\left[\left(X_{n}-\hat{X}_{n}-Z_{n}\right)^{2}\right]$ is obtained by noting that

$$
\mathrm{E}\left[\left(X_{n}-\hat{X}_{n}-Z_{n}\right)^{2}\right]=\theta^{2} \mathrm{E}\left[\left(Z_{n-1}-\mathrm{P}\left(Z_{n-1} \mid X_{1}, \ldots, X_{n-1}\right)\right)^{2}\right] .
$$

Equation (3.8) provides

$$
\mathrm{P}\left(Z_{n-1} \mid X_{1}, \ldots, X_{n-1}\right)=X_{n-1}-\phi X_{n-2}+\theta \mathrm{P}\left(Z_{n-2} \mid X_{1}, \ldots, X_{n-1}\right)
$$

hence,

$$
\mathrm{E}\left[\left(X_{n}-\hat{X}_{n}-Z_{n}\right)^{2}\right]=\theta^{4} \mathrm{E}\left[\left(Z_{n-2}-\mathrm{P}\left(Z_{n-2} \mid X_{1}, \ldots, X_{n-1}\right)\right)^{2}\right] .
$$

We recurse these arguments and use $\mathrm{E}\left[\left(Z_{0}-\mathrm{P}\left(Z_{0} \mid X_{1}, \ldots, X_{n-1}\right)\right)^{2}\right] \leq \sigma^{2}$, to get $\mathrm{E}\left[\left(X_{n}-\hat{X}_{n}-Z_{n}\right)^{2}\right] \leq \theta^{2 n} \sigma^{2}$. Using this and $v_{n} \geq \sigma^{2}, v_{\infty}=\sigma^{2}$, and $|\theta| \leq 1$ in (4.9) gives

$$
\left|\theta_{n, 1}-\theta\right| \leq \sqrt{2}|\theta|^{n}+\frac{|\theta|\left(v_{n-1}-v_{\infty}\right)}{\sigma^{2}} .
$$

To bound $\left(v_{n-1}-v_{\infty}\right) / \sigma^{2}$, we use the DHR shape established in Proposition 3.1, (4.4), $M_{\infty}=\theta^{2}<1, \gamma(0)=\sigma^{2}\left(1+2 \phi \theta+\theta^{2}\right)\left(1-\phi^{2}\right)^{-1}$, and some algebra, to obtain

$$
\frac{v_{n-1}-v_{\infty}}{\sigma^{2}} \leq \frac{\gamma(0) \alpha^{2}(n-1) M_{\infty}}{\left(1-M_{\infty}\right) \sigma^{2}} \leq \frac{\theta^{2 n-2}(1+\phi \theta)^{2}}{\left(1-\phi^{2}\right)\left(1-\theta^{2}\right)} .
$$

Substituting (4.11) into (4.10) produces the convergence rate quoted in (4.6). 


\section{Acknowledgement}

Support from the National Science Foundation, grant number DMS 0304407, is acknowledged.

\section{References}

Berenhaut, K. S. And Lund, R. B. (2001). Geometric renewal convergence rates from hazard rates. J. Appl. Prob. 38, 180-194.

Berenhaut, K. S. And Lund, R. B. (2002). Renewal convergence rates for DHR and NWU lifetimes. Prob. Eng. Inf. Sci. 16, 67-84.

Billingsley, P. (1995). Probability and Measure, 3rd edn. John Wiley, New York.

Brockwell, P. J. And Davis, R. A. (1991). Time Series: Theory and Methods, 2nd edn. Springer, New York.

Brown, M. (1980). Bounds, inequalities, and monotonicity properties for some specialized renewal processes. Ann. Prob. 8, 227-240.

Hansen, B. G. And Frenk, J. B. G. (1991). Some monotonicity properties of the delayed renewal function. J. Appl. Prob. 28, 811-821.

KiJima, M. (1997). Markov Processes for Stochastic Modeling. Chapman \& Hall, London.

LiggetT, T. (1989). Total positivity and renewal theory. In Probability, Statistics, and Mathematics, eds T. W. Anderson, K. B. Athreya and D. L. Iglehart, Academic Press, Boston, MA, pp. 141-162.

Lund, R. B., Zhao, Y. And Kiessler, P. C. (2006). A monotonicity in reversible Markov chains. J. Appl. Prob. 43, 486-499.

Müller, A. And Stoyan, D. (2002). Comparison Methods for Stochastic Models and Risks. John Wiley, New York. Pourahmadi, M. (2001). Foundations of Time Series Analysis and Prediction Theory. John Wiley, New York.

Shaked, M. And Shanthikumar, J. G. (1994). Stochastic Orders and Their Applications. Academic Press, New York. 